\title{
Multiplicative Gain Changes Are Induced by Excitation or Inhibition Alone
}

\author{
Brendan K. Murphy ${ }^{1}$ and Kenneth D. Miller ${ }^{1,2}$ \\ ${ }^{1}$ Graduate Group in Biophysics, W. M. Keck Center for Integrative Neuroscience, and 2Departments of Physiology and Otolaryngology, Sloan-Swartz Center \\ for Theoretical Neurobiology, University of California, San Francisco, California 94143-0444
}

\begin{abstract}
We model the effects of excitation and inhibition on the gain of cortical neurons. Previous theoretical work has concluded that excitation or inhibition alone will not cause a multiplicative gain change in the curve of firing rate versus input current. However, such gain changes in vivo are measured in the curve of firing rate versus stimulus parameter. We find that when this curve is considered, and when the nonlinear relationships between stimulus parameter and input current and between input current and firing rate in vivo are taken into account, then simple excitation or inhibition alone can induce a multiplicative gain change. In particular, the power-law relationship between voltage and firing rate that is induced by neuronal noise is critical to this result. This suggests an unexpectedly simple mechanism that may underlie the gain modulations commonly observed in cortex. More generally, it suggests that a smaller input will multiplicatively modulate the gain of a larger one when both converge on a common cortical target.
\end{abstract}

Key words: multiplication; gain modulation; noise; power-law; cortex; tuning curve

\section{Introduction}

Gain modulation is a roughly multiplicative or divisive change in the tuning curve of a neuron to one stimulus parameter as some other parameter or state is modified. Such gain changes are observed frequently in the responses of cortical neurons and are thought to play an important role in neural computations (for review, see Salinas and Thier, 2000). A particularly well studied example exists in monkey posterior parietal cortex, in which the responses of neurons to the retinal position of a visual stimulus are multiplicatively scaled by eye position (Andersen and Mountcastle, 1983; Andersen et al., 1985). Similar modulation of responses by eye position is seen in a variety of visual areas (Galletti and Battaglini, 1989; Boussaoud et al., 1993; Bremmer et al., 1997b; Trotter and Celebrini, 1999). This gain modulation has been proposed to underlie coordinate transforms computed by these neurons, which are necessary for visually guided reaching (Zipser and Andersen, 1988; Salinas and Abbott, 1995, 2001; Pouget and Sejnowski, 1997; Pouget and Snyder, 2000). Gain modulations have also been observed in the enhancement of neural responses by attention. McAdams and Maunsell (1999a) showed that attention can increase the gain of the orientation tuning curves of neurons in areas V1 and V4 of macaque visual cortex, whereas Treue and Martinez-Trujillo (1999) found that attention increases the gain of direction tuning curves in macaque area MT. Modulation of responses in V1 by stimuli outside the classical receptive field seems to be divisive in character $(\mathrm{Ca}-$

\footnotetext{
Received April 25, 2003; revised Sept. 10, 2003; accepted Sept. 12, 2003.

This work was supported by National Institutes of Health Grant R01-EY11001 to K.D.M. and by a predoctoral fellowship to B.K.M. from a Burroughs-Wellcome Institutional Award at the Scientific Interface to the Program in Quantitative Biology at University of California, San Francisco.

Correspondence should be addressed to Kenneth Miller, Department of Physiology, University of California, San Francisco, 513 Parnassus, San Francisco, CA 94143-0444. E-mail: ken@phy.ucsf.edu. Copyright $\odot 2003$ Society for Neuroscience $\quad$ 0270-6474/03/2310040-12\$15.00/0
}

vanaugh et al., 2002; Palmer and Nafziger, 2002; Muller et al., 2003). Gain modulation can also be induced pharmacologically. Fox et al. (1990) found that by iontophoretically applying NMDA to neurons in cat V1, they could increase the gain of the contrastresponse (CR) curve of the neuron.

Despite the apparent importance of multiplicative gain modulation in the cortex, the mechanisms responsible for producing such gain changes are not well understood (but, see Srinivasan and Bernard, 1976; Fox and Daw, 1992; Mel, 1993; Salinas and Abbott, 1996; Chance et al., 2002; Doiron et al., 2002; Smith et al., 2002). In particular, it has been concluded that simple excitation or inhibition alone cannot achieve a gain change (Holt and Koch, 1997; Chance et al., 2002), except at low firing rates (Doiron et al., 2001). Instead, it has been shown recently that concurrent, balanced increases in background excitation and inhibition together, which cause an increase in current noise and in conductance with no net depolarization or hyperpolarization, can serve to divisively decrease gain (Chance et al., 2002). These conclusions were based on examining the gain of the relationship between injected current and firing rate. However, multiplicative gain changes in cortex in vivo are observed in the relationship between a "stimulus parameter" and firing rate. Here, we consider the nonlinear relationship between stimulus parameter and injected current, as well as the nonlinear relationship between injected current and firing rate. We show that multiplicative gain changes arise robustly from the simple addition of excitation or inhibition alone, provided the modulating excitation or inhibition is small relative to the peak of the tuning curve of the driving excitation. That is, the observed cortical gain changes can be induced if the modulating influence simply adds or subtracts excitation or inhibition.

An important part of our model is the large background synaptic conductances to which neurons are subject in vivo, which 
give rise to a noisy subthreshold membrane potential (Destexhe and Paré, 1999; Anderson et al., 2000). A noisy subthreshold membrane potential in turn gives rise to an expansive power-law relationship between the average membrane potential and the firing rate of a neuron (Hansel and van Vreeswijk, 2002; Miller and Troyer, 2002). This nonlinear relationship between voltage or input current and firing rate, along with the nonlinear relationship between stimulus parameter and input current, together cause excitation or inhibition alone to yield roughly multiplicative gain changes in neuronal responses. We demonstrate this using both numerical simulations and a simple analytical model.

\section{Materials and Methods}

We simulate a cortical neuron using two models: a conductance-based, integrate-and-fire model and a Hodgkin-Huxley-type model.

Integrate-and-fire model. The integrate-and-fire model is described by the following equation:

$$
C \frac{\mathrm{d} V}{\mathrm{~d} t}=g_{\text {leak }}\left(E_{\text {leak }}-V\right)+\sum_{\mathrm{i}} g_{\mathrm{i}}\left(E_{\mathrm{i}}-V\right)+I_{\text {inj }},
$$

where $C$ is the capacitance, $g_{\text {leak }}$ is the leak conductance and $E_{\text {leak }}$ is its reversal potential, $I_{\mathrm{inj}}$ is the injected current, and the $g_{\mathrm{i}}$ are conductances with corresponding reversal potentials $E_{\mathrm{i}}$. When the voltage reaches the spike threshold $V_{\text {thresh }}$, it is reset to $V_{\text {reset }}$ and held there for a refractory period $\left(t_{\text {refract }}\right)$.

The parameters for the integrate-and-fire model and its synaptic and noise currents were selected to match cortical properties, primarily in the course of previous work (Troyer and Miller, 1997; Troyer et al., 1998; Krukowski and Miller, 2001; Palmer and Miller, 2002) but with minor adjustments in the present work. In particular, the parameters were designed without reference to (and before obtaining) the results presented here. The values for the following parameters are the same in each simulation: $g_{\text {leak }}=10 \mathrm{nS}, C=488 \mathrm{pF}, E_{\text {leak }}=-70 \mathrm{mV}, V_{\text {thresh }}=-54 \mathrm{mV}$, $V_{\text {reset }}=-60 \mathrm{mV}$, and $t_{\text {refract }}=1.7 \mathrm{msec}$. The value of $C$ was chosen so that, after taking into account additional background (noise) conductances described below, the resting membrane time constant is $20 \mathrm{msec}$. This is consistent with values of 15-24 msec observed in vivo for cortical neurons (Hirsch et al., 1998).

There are two excitatory synaptic conductances, NMDA and AMPA, and two inhibitory synaptic conductances, $\mathrm{GABA}_{\mathrm{A}}$ and $\mathrm{GABA}_{\mathrm{B}}$. Their reversal potentials are $E_{\mathrm{NMDA}}=0 \mathrm{mV}, E_{\mathrm{AMPA}}=0 \mathrm{mV}, E_{\mathrm{GABA}_{\mathrm{A}}}=-70$ $\mathrm{mV}$, and $E_{\mathrm{GABA}_{\mathrm{B}}}=-90 \mathrm{mV}$. The NMDA conductance is voltage dependent in accordance with the model described by Jahr and Stevens (1990), using $\left[\mathrm{Mg}^{2+}\right]=1.2 \mathrm{~mm}$. The voltage we use to compute the NMDA conductance is the "shadow voltage" $V_{s}$. $V_{\mathrm{s}}$ is obtained by integrating the membrane potential continuously in time in the absence of a spike threshold (e.g., it is not reset when it reaches the spike threshold). This is meant to approximate the potential experienced by NMDA channels located on the dendrites of the neuron and to eliminate discontinuities in the conductance at spike times. This model yields an NMDA conductance that is $3.7 \%$ of maximum at the resting potential of the neuron (where maximum is defined to be the conductance at a voltage of 100 $\mathrm{mV}$ ) and $10.6 \%$ of maximum when $V_{\mathrm{s}}=-51 \mathrm{mV}$ (the largest in this study).

The time courses of AMPA, GABA $A$, and $\mathrm{GABA}_{B}$ conductances after presynaptic action potentials are modeled as a difference of single exponentials:

$$
g(t)=\sum_{\Delta t_{j}} \bar{g}\left(e^{-\Delta t_{j} / \tau^{\text {fall }}}-e^{-\Delta t_{j} / \tau^{\text {rise }}}\right) .
$$

Here, $\Delta t_{\mathrm{j}}$ is defined as $\left(t-t_{\mathrm{j}}\right)$, where $t_{\mathrm{j}}$ is the time of the $\mathrm{jth}$ presynaptic action potential and $t_{\mathrm{j}}<\mathrm{t}$. The time constants are $\tau_{\mathrm{AMPA}}^{\text {rise }}=0.25 \mathrm{msec}$, $\tau_{\mathrm{AMPA}}^{\text {fall }}=1.75 \mathrm{msec}, \tau_{\mathrm{GABA}_{\mathrm{A}}}^{\text {rise }}=0.75 \mathrm{msec}, \tau_{\mathrm{GABA}_{\mathrm{A}}}^{\text {fall }}=5.25 \mathrm{msec}, \tau_{\mathrm{GABA}_{\mathrm{B}}}^{\text {rise }}=$ $40 \mathrm{msec}$, and $\tau_{\mathrm{GABA}_{\mathrm{B}}}^{\text {fall }}=80 \mathrm{msec}$. Parameters for GABAergic synaptic conductances are set to roughly match experimental data (Connors et al.,
1988; Benardo, 1994). NMDA conductances decay as a double exponential with a fast and slow component:

$$
g\left(t, V_{s}\right)=\sum_{\Delta t_{j}} \bar{g}\left(V_{s}\right)\left(f_{\text {fast }} e^{-\Delta t_{j} / f_{\text {fast }}^{\text {fall }}}+f_{\text {slow }} e^{-\Delta t_{j} / \tau_{\text {slow }}^{\text {fall }}}-e^{-\Delta t_{j} / \tau^{\text {rise }}}\right) .
$$

Parameters for NMDA conductances are taken from experimental data for adult rats (Carmignoto and Vicini, 1992): $\tau_{\text {fast }}^{\text {fall }}=63 \mathrm{msec}, \Delta_{\text {slow }}^{\text {fall }}=200$ msec, $f_{\text {fast }}=0.88$, and $f_{\text {slow }}=\left(1-f_{\text {fast }}\right)$. We set $\tau_{\mathrm{NMDA}}^{\text {rise }}=5.5 \mathrm{msec}$ to match the experimentally observed $10-90 \%$ rise time for NMDA receptor-mediated postsynaptic currents (Lester et al., 1990).

The sizes of synaptic conductances evoked by presynaptic action potentials are set in terms of their total conductance integrated over time in units of nS·msec. The relative strengths of AMPA and NMDA conductances are set to match those observed in thalamocortical slices (Crair and Malenka, 1995). This, along with $\left[\mathrm{Mg}^{2+}\right]=1.2 \mathrm{~mm}$, yields the result that, at $V_{\text {thresh }}$, the time integrated conductance for NMDA is 2.57 times that of AMPA. The AMPA conductance evoked by a single excitatory presynaptic action potential is set to $2.8 \mathrm{nS} \cdot \mathrm{msec}$. At $V_{\text {thresh }}$, the evoked NMDA conductance is $7.2 \mathrm{nS} \cdot \mathrm{msec}$. The $\mathrm{GABA}_{\mathrm{A}}$ conductance resulting from a single inhibitory presynaptic action potential is set to $8 \mathrm{nS} \cdot \mathrm{msec}$, and the $\mathrm{GABA}_{\mathrm{B}}$ conductance to $2 \mathrm{nS} \cdot \mathrm{msec}$.

In addition to the above described synaptic conductances, the model includes two fluctuating background conductances, an inhibitory conductance $\left[g_{\mathrm{I}}(t)\right]$ with $E_{\mathrm{rev}}=-80 \mathrm{mV}$ and an excitatory conductance $\left[g_{\mathrm{E}}(t)\right]$ with $E_{\mathrm{rev}}=0 \mathrm{mV}$. These conductances are meant to simulate the background synaptic input received by cortical neurons in vivo. They are produced by an Ornstein-Uhlenbeck process, as described by Destexhe et al. (2001):

$$
\frac{\mathrm{d} g(t)}{\mathrm{d} t}=\frac{g_{0}-g(t)}{\tau}+\chi(t) \sqrt{\frac{\sigma^{2}}{\tau}}
$$

where $g_{0}$ is the mean conductance, $\tau$ is a noise time constant, $\sigma^{2}$ is the variance of the conductance, and $\chi(t)$ is a Gaussian random variable with 0 mean and a SD of 1 . Parameters were chosen by beginning with the parameters used by Palmer and Miller (2002) and adjusting these to produce membrane potential fluctuations of $\sim 5 \mathrm{mV}$ at rest and a rest potential of approximately $-70 \mathrm{mV}$. This is in accordance with recordings from cortical cells in vivo (Anderson et al., 2000). For the inhibitory background conductance, $g_{0}=12.0 \mathrm{nS}, \tau=34.1 \mathrm{msec}$, and $\sigma=4.3 \mathrm{nS}$. For the excitatory background conductance, $\mathrm{g}_{0}=2.4 \mathrm{nS}, \tau=34.1 \mathrm{msec}$, and $\sigma=2.4 \mathrm{nS}$. The noise time constant of $34.1 \mathrm{msec}$ was chosen to cause the power spectrum of the voltage noise to match that seen in experimental data generously provided by Jeff Anderson and David Ferster (Northwestern University, Evanston, IL) (this matching was performed by S. E. Palmer in the laboratory of K.D.M.) (Palmer and Miller, 2002). The mean background conductance, combined with $g_{\text {leak }}$, gives the cell a resting input resistance $(R)$ of $41 \mathrm{M} \Omega$. The capacitance $(C)$ is set to give the cell a resting membrane time constant $\left(\tau_{\mathrm{m}}=R C\right)$ of $20 \mathrm{msec}$.

Hodgkin-Huxley model. Simulations of a more biophysically detailed single compartment model were produced using the NEURON simulation environment (Hines and Carnevale, 1997). This model includes fluctuating background conductances produced by an Ornstein-Uhlenbeck process, voltage-dependent sodium and potassium conductances to model action potentials, and a noninactivating potassium conductance responsible for spike frequency adaptation as described by Destexhe et al. (2001) for a single compartment neuron model. Parameters for the model were taken from Destexhe et al. (2001): $g_{\text {leak }}=0.045 \mathrm{mS} / \mathrm{cm}^{2}$, $E_{\text {leak }}=-80 \mathrm{mV}, C=1 \mu \mathrm{F} / \mathrm{cm}^{2}$. For the inhibitory background conductance, $g_{0}=57.0 \mathrm{nS}, \tau=10.5 \mathrm{msec}$, and $\sigma=15.84 \mathrm{nS}$. For the excitatory background conductance, $g_{0}=12 \mathrm{nS}, \tau=2.7 \mathrm{msec}$, and $\sigma=7.2 \mathrm{nS}$. The densities of voltage-dependent sodium and potassium channels are 480 $\mathrm{pS} / \mu \mathrm{m}^{2}$ and $100 \mathrm{pS} / \mu \mathrm{m}^{2}$, respectively. The density of spike adaptation potassium channels is $3 \mathrm{pS} / \mu \mathrm{m}^{2}$. The surface area of the simulated neuron is $34,636 \mu \mathrm{m}^{2}$.

The model also includes a hyperpolarization-activated conductance $\left(I_{\mathrm{h}}\right)$ with kinetics modeled as described by Migliore (2003). This conduc- 
tance has a reversal potential of $-43 \mathrm{mV}$ (Stuart and Spruston, 1998) and a density of $0.05 \mathrm{mS} / \mathrm{cm}^{2}$.

\section{Results}

Our results are divided into three sections. First, we present the results of a series of numerical simulations of a single model neuron of the visual cortex. The model neuron is a conductancebased integrate-and-fire neuron. Its input includes noise conductances designed to match voltage noise observed in vivo (Anderson et al., 2000), which make the RMS voltage noise $\sim 5 \mathrm{mV}$. In the second section, we provide a simplified, but more intuitive, analytical model of the neuron that shows the generality and robustness of the results obtained with the integrate-and-fire model. Finally, to further illustrate the robustness of the results, we show that the assumptions of the analytical model and the results of the integrate-and-fire model all hold for a more biophysically detailed Hodgkin-Huxley-type model neuron that also receives noise conductances. This model includes conductances responsible for spike generation and spike frequency adaptation, as well as a subthreshold hyperpolarization-activated conductance $\left(I_{\mathrm{h}}\right)$. We refer to the two numerical models as either the integrate-and-fire model or the Hodgkin-Huxley model.

Gain is defined here as the slope of a plot of a stimulus parameter, such as contrast or orientation, versus the response of the neuron. A pure gain change is one in which the curve of response versus stimulus parameter is multiplicatively scaled, so that the gain is scaled by a constant factor for all values of the stimulus parameter.

\section{Integrate-and-fire simulations}

\section{Contrast-response curve gain}

Neurons in the visual cortex respond to stimuli of increasing contrast with an increasing firing rate. Plots of contrast versus firing rate are often well fit by a hyperbolic ratio function (Albrecht and Hamilton, 1982; Sclar et al., 1990):

$$
R=R_{\max }\left(\frac{C^{n}}{C^{n}+C_{50}^{n}}\right)+S,
$$

where $C$ is the contrast, $R$ is the firing rate, $S$ is the spontaneous activity, and $C_{50}$ is the contrast that gives a half-maximal stimulus-induced firing rate. We refer to a plot of contrast versus firing rate as a CR curve. The gain of the neuron is the slope of the CR curve.

We assume that our model neuron receives a stimulus-driven excitatory Poisson input. The rate of this input is a hyperbolic ratio function of stimulus contrast, with $R_{\max }=2000 \mathrm{~Hz}, C_{50}=$ $0.133, n=1.2$, and $S=0$. This is designed to model synaptic input from a previous stage of visual processing. We then study how the gain of the response of the model neuron to the stimulus is altered by additional excitation or inhibition in the form of glutamate or GABA receptor-binding drugs or direct injected current.

Iontophoresis. We modeled iontophoretic application of drugs binding to NMDA, AMPA, GABA $\mathrm{A}_{\mathrm{A}}$, or $\mathrm{GABA}_{\mathrm{B}}$ receptors by opening a constant conductance of the appropriate type and studied the effects on the CR curve of the neuron (Fig. 1A).

We first simulated the iontophoresis of NMDA onto the neuron by opening a constant NMDA conductance equivalent to 10 $\mathrm{nS}$ if the neuron were held at $+100 \mathrm{mV}$. Because of the voltage dependence of NMDA channels, the mean iontophoretic NMDA conductance is $0.48 \mathrm{nS}$ at zero contrast and increases to $1.1 \mathrm{nS}$ at maximum contrast. This conductance increased the firing rate of
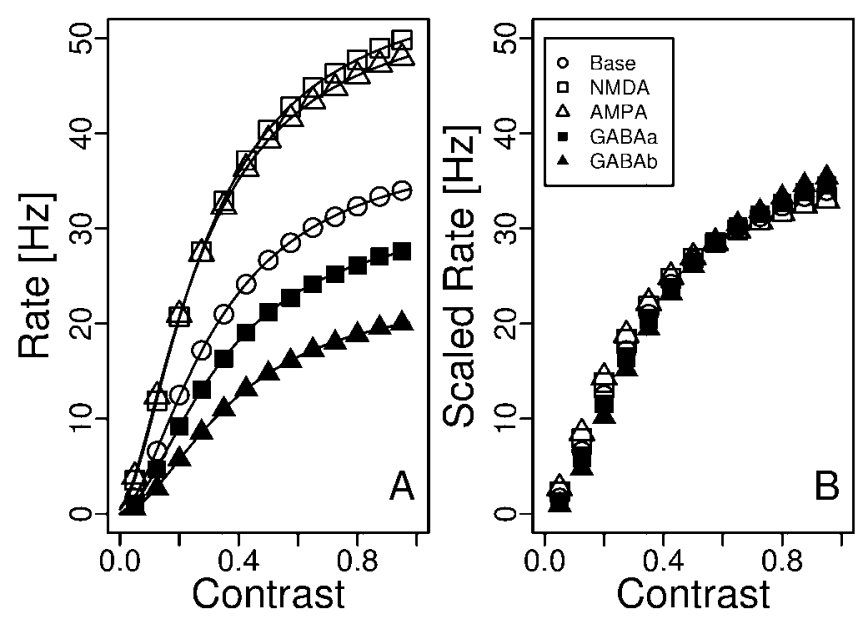

Figure 1. A, Plot of contrast versus firing rate for the model neuron without drugs (open circles), and with iontophoretic NMDA (open squares), AMPA (open triangles), GABA (closed $^{\circ}$ squares), or $\mathrm{GABA}_{B}$ (closed triangles) applied. Each data point represents an average of $2060 \mathrm{sec}$ trials. Solid lines are fits of the data to a hyperbolic ratio function (Eq. 5). Parameters for the fits were obtained using a nonlinear least squares algorithm and are shown in Table 1. B, Curves from $A$ scaled to optimally (least squares) fit the Base curve.

the model neuron at all contrasts. The maximum firing rate (at $C=1.0$ ) increased to $50 \mathrm{~Hz}$ from $34 \mathrm{~Hz}$ in the baseline curve. The firing rate at $C=0.0$ increased to $0.96 \mathrm{~Hz}$ from $0.26 \mathrm{~Hz}$. This corresponds to a $46 \%$ increase in the average slope of the CR curve. One might imagine that this slope increase is caused by the voltage-dependent increase of the NMDA conductance. However, when we simulated the iontophoresis of AMPA by opening a constant $1.0 \mathrm{nS}$ AMPA conductance, we observed a similar increase in firing rates. The maximum firing rate increased to 48 $\mathrm{Hz}$, and the firing rate at $\mathrm{C}=0.0$ increased to $0.81 \mathrm{~Hz}$. The average slope of the CR curve increased by $40 \%$. Because the AMPA conductance is not voltage dependent, this implies another mechanism of gain change.

We next simulated the iontophoresis of inhibitory drugs binding to $\mathrm{GABA}_{\mathrm{A}}$ or $\mathrm{GABA}_{\mathrm{B}}$ receptors. In both cases, the firing rate of the model neuron was reduced at all contrasts. A constant $2.0 \mathrm{nS}$ $\mathrm{GABA}_{\mathrm{A}}$ conductance decreased the maximum firing rate from 34 to $28 \mathrm{~Hz}$ and the firing rate at $C=0.0$ from 0.26 to $0.15 \mathrm{~Hz}$. The average slope of the CR curve decreased by $17 \%$. A constant 2.0 $\mathrm{nS} \mathrm{GABA} \mathrm{B}_{\mathrm{B}}$ conductance had a larger effect, decreasing the maximum firing rate to $20 \mathrm{~Hz}$ and the firing at $C=0.0$ to $0.06 \mathrm{~Hz}$. The average slope decreased by $41 \%$. The different effects of $\mathrm{GABA}_{\mathrm{A}}$ and $\mathrm{GABA}_{\mathrm{B}}$ can be attributed to their different reversal potentials.

We scaled the iontophoretic CR curves to optimally fit the baseline curve (Fig. 1B). The overlap of the scaled CR curves indicates that changes in the firing rate caused by iontophoretic conductances are very close to pure, multiplicative gain changes. Nonetheless, there are clearly systematic deviations from a purely multiplicative scaling. These deviations are made clear by analyzing the fits of the CR curves to hyperbolic ratio functions (Fig. $1 \mathrm{~A}$, solid lines). Fit parameters are given in Table 1. The most significant of these deviations are left and right shifts of the CR curve, indicated by changes in $C_{50}$ (Eq. 5). NMDA and AMPA conductances shifted the baseline curve left, decreasing $C_{50}$ by 11 and $13 \%$, respectively. $\mathrm{GABA}_{\mathrm{A}}$ and $\mathrm{GABA}_{\mathrm{B}}$ shifted the curve right, increasing the baseline $C_{50}$ by 6 and 17\%, respectively. NMDA and AMPA also increased spontaneous activity $(C=0.0)$ somewhat, whereas $\mathrm{GABA}_{\mathrm{A}}$ and $\mathrm{GABA}_{\mathrm{B}}$ reduced it. 
Table 1. Values of the fit parameters for the solid lines in Figure $1 A$ and the scale factors used in Figure $1 B$

\begin{tabular}{llllll}
\hline & $R_{\max }$ & $C_{50}$ & $n$ & $S$ & Scale \\
\hline Base & 39.5 & 0.325 & 1.66 & 0.0600 & 1.00 \\
NMDA & 56.5 & 0.290 & 1.61 & 0.452 & 1.50 \\
AMPA & 54.1 & 0.280 & 1.58 & 0.632 & 1.46 \\
GABA $_{A}$ & 32.5 & 0.346 & 1.71 & -0.0294 & 0.800 \\
GABA $_{B}$ & 24.0 & 0.381 & 1.78 & -0.0760 & 0.565 \\
\hline
\end{tabular}

Parameters are defined in Equation 5.
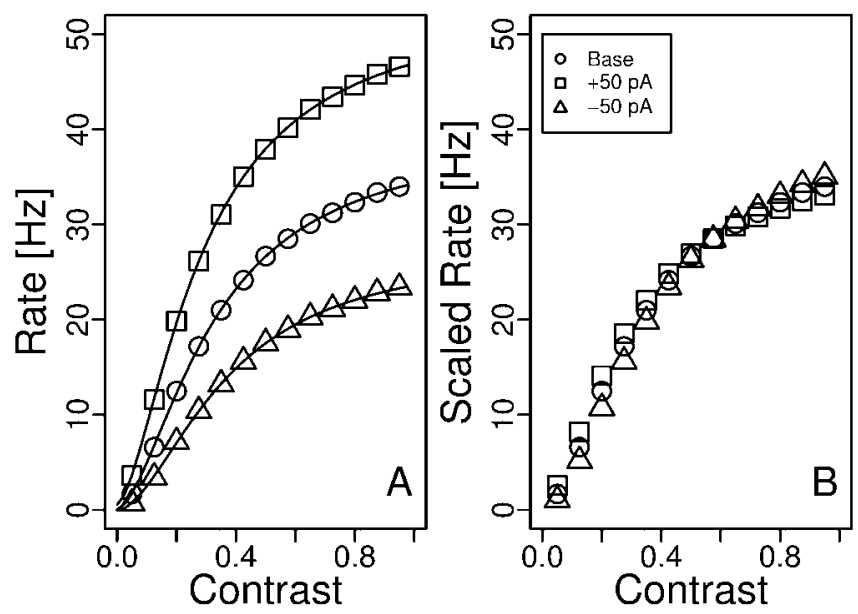

Figure 2. $A$, Plot of contrast versus firing rate for the model neuron without injected current (circles) and with $+50 \mathrm{pA}$ (squares) or $-50 \mathrm{pA}$ (triangles) of current injected. Each data point represents an average of $2060 \mathrm{sec}$ trials. Solid lines are fits of the data to a hyperbolic ratio function (Eq. 5). Parameters for the fits are shown in Table 2. B, Curves from A scaled to optimally (least squares) fit the Base curve.

Injected current. In our model, iontophoresis causes both polarizing ionic current and changes in conductance. The conductance changes are small relative to the mean resting conductance of the neuron of $\sim 24 \mathrm{nS}$, rendering it unlikely that the conductance changes are a significant cause of the gain change. However, to directly separate the effects of injected current and conductance change, we next simulated the direct injection of current into the model neuron.

The effects of current injection on CR curves are very much like the effects of iontophoretic drugs (Fig. 2). Injection of depolarizing current into the neuron has effects similar to an iontophoretic AMPA or NMDA conductance. Current (50 pA) increased the maximum firing rate from 34 to $47 \mathrm{~Hz}$ and the firing rate at $C=0.0$ from 0.26 to $0.73 \mathrm{~Hz}$. The average slope of the $\mathrm{CR}$ curve increased by $37 \%$. Conversely, injection of the hyperpolarizing current has effects similar to an iontophoretic $\mathrm{GABA}_{\mathrm{A}}$ or $\mathrm{GABA}_{\mathrm{B}}$ conductance. Current $(-50 \mathrm{pA})$ decreased the maximum firing rate to $24 \mathrm{~Hz}$ and the firing rate at $C=0.0$ to $0.09 \mathrm{~Hz}$. The average slope of the CR curve decreased by $29 \%$.

The changes in firing rate caused by injected current are close to purely multiplicative gain changes (Fig. $2 B$ ). Deviations from purely multiplicative changes are indicated by the fit parameters in Table 2. These deviations are in the same direction and of similar magnitude to those seen above for iontophoresis. Positive current shifted the baseline CR curve left, decreasing $C_{50}$ by $12 \%$. Negative current shifted the curve right, increasing the baseline $C_{50}$ by $12 \%$. These results imply that polarizing current, and not a change in conductance, is the primary factor responsible for changing the gain of the model neuron.

Our model of the effects of injecting current can be directly
Table 2. Values of the fit parameters for the solid lines in Figure $2 A$ and the scale factors used in Figure $2 B$

\begin{tabular}{llllll}
\hline & $R_{\max }$ & $C_{50}$ & $n$ & $S$ & Scale \\
\hline Base & 39.5 & 0.325 & 1.66 & 0.0600 & 1.00 \\
$+50 \mathrm{pA}$ & 52.7 & 0.285 & 1.59 & 0.536 & 1.41 \\
$-50 \mathrm{pA}$ & 27.7 & 0.365 & 1.76 & -0.0751 & 0.667 \\
\hline
\end{tabular}

Parameters are defined in Equation 5.
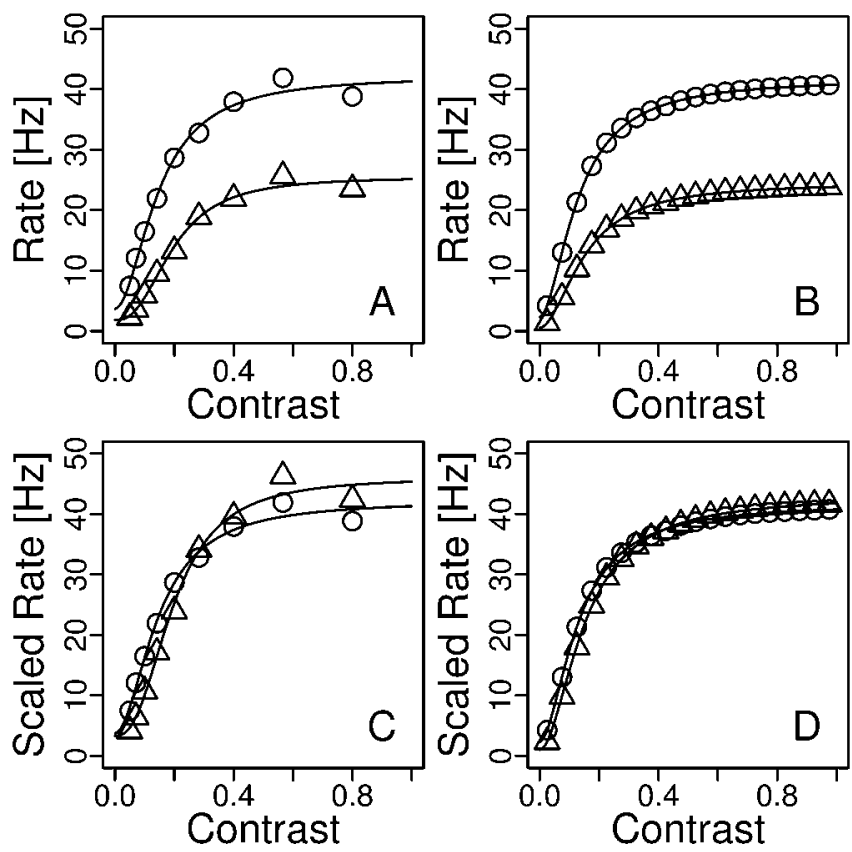

Figure 3. A, Replotted data from Sanchez-Vives et al. (2000, their Fig. 12A), demonstrating the effects of hyperpolarizing injected current on the $C R$ functions of neurons in primary visual cortex. Circles, Curves with no injected current; triangles, curves with hyperpolarizing current injected. $B, 0$ ur simulated CR curves. Control curve (circles) uses input parameters $R_{\max }=1400$ $\mathrm{Hz}, \mathrm{C}_{50}=0.08, n=1.6$, and $\mathrm{S}=600 \mathrm{~Hz}$, designed to reproduce control curve in $A$. Curve with hyperpolarizing current (triangles) used current of $-90 \mathrm{pA}$, designed to produce a similar reduction of $R_{\max }$ as in $A$. Each data point represents an average of 2060 sec trials. C, D, Plots from $A$ and $B$ with the hyperpolarized curve scaled to optimally (least squares) match the control curve. Data in $A$ are averages over multiple cells studied over four octaves of contrast; we have plotted the four octaves as $5-80 \%$. Solid lines are fits of the data to a hyperbolic ratio function (Eq. 5). Fit parameters for the model are $R_{\max }=39 \mathrm{~Hz}, \mathrm{C}_{50}=0.13, n=1.8$, and $\mathrm{S}=2.3 \mathrm{~Hz}$ without and $R_{\max }=24 \mathrm{~Hz}, C_{50}=0.15, n=1.9$, and $S=0.6 \mathrm{~Hz}$ with hyperpolarizing current. Fit parameters for the experimental data are $R_{\max }=38 \mathrm{~Hz}, \mathrm{C}_{50}=0.15, n=1.9$, and $S=3.7$ $\mathrm{Hz}$ without and $R_{\max }=24 \mathrm{~Hz}, \mathrm{C}_{50}=0.2, n=2.5$, and $S=1.8 \mathrm{~Hz}$ with hyperpolarizing current.

compared with existing in vivo data. Sanchez-Vives et al. (2000) examined the effect on visual cortical CR curves of injecting a hyperpolarizing current into the cell (Fig. $3 A$ ). Effects in the model are strikingly similar (Fig. $3 B$ ). The simulation produces a more purely multiplicative effect than is seen in the average experimental data (Fig. 3D), but the deviations are in the same directions in simulation as in the data. In particular, the hyperpolarization induces a statistically significant (Sanchez-Vives et al., 2000) increase in $C_{50}$ in the experimental data, as predicted by the model. It should be noted that the experimental data are averaged over many cells; if each cell were modulated multiplicatively but different cells had different CR curves and were modulated by different factors, then the modulation of the average would not be purely multiplicative. Thus, it is possible that individual cells show a more purely multiplicative effect than the average data. In any case, the overall resemblance suggests that 

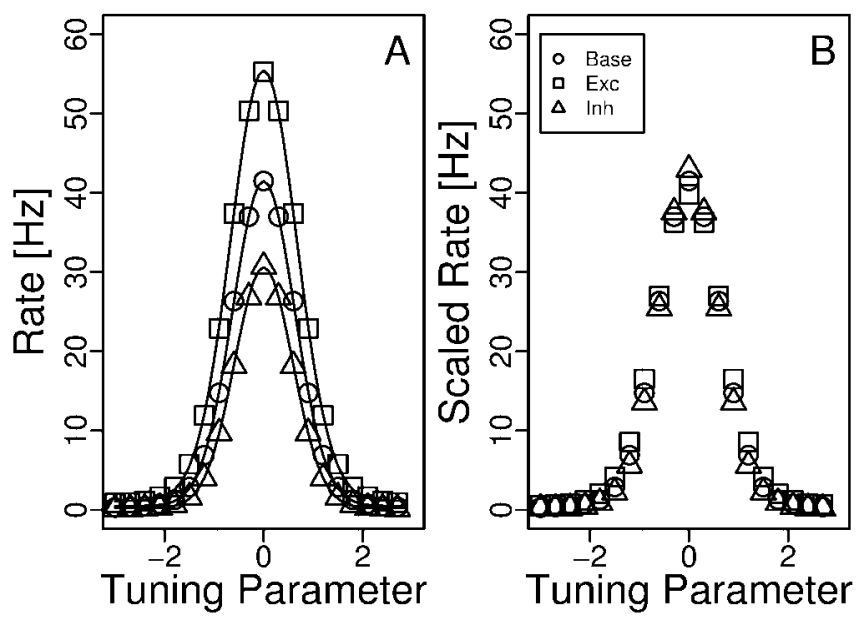

Figure 4. A, Plot of the tuning parameter in Equation 6 versus firing rate for the model neuron without modulatory input (circles) and with a modulatory $250 \mathrm{~Hz}$ excitatory (squares) or $250 \mathrm{~Hz}$ inhibitory (triangles) Poisson input. Each data point represents an average of $2060 \mathrm{sec}$ trials. Solid lines are fits of the data to a Gaussian function (Eq. 6). Parameter values for the fits are shown in Table 3. $B$, Curves from $A$ scaled to optimally (least squares) fit the Base curve.

our simple model gives a reasonable representation of neurons in vivo.

Tuning curve gain

A number of influences, including attention and eye position, have been shown to change the gain of tuning curves in cortex. Although the mechanisms by which these gain changes occur are not clear, we demonstrate that, in our model neuron, one synaptic input can modulate the gain of the response to another. To do so, we introduce two Poisson inputs to the neuron. The first is an excitatory driving input, the rate $R$ of which is a Gaussian function of an arbitrary stimulus parameter $\theta$ :

$$
R(\theta)=R_{\max } \exp \left(-\frac{\theta^{2}}{2 \sigma^{2}}\right)+S .
$$

The second is a modulatory input, either excitatory or inhibitory, the rate of which is independent of $\theta$. For the driving input, $\sigma=$ $1.0, R_{\max }=2000 \mathrm{~Hz}$, and $S=0 \mathrm{~Hz}$.

We constructed tuning curves for the model neuron by plotting average firing rate versus $\theta$, with and without modulatory inputs (Fig. 4A). The driving input alone produced a maximum firing rate at $\theta=0.0$ of $41 \mathrm{~Hz}$ and a minimum firing rate at $\theta=$ \pm 3 of $0.29 \mathrm{~Hz}$. We then added modulatory inputs to the neuron. A $250 \mathrm{~Hz}$ excitatory input increased the firing rate of the neuron for all values of $\theta$. The maximum response increased to $55 \mathrm{~Hz}$, and the firing rate at $\theta= \pm 3$ increased to $0.86 \mathrm{~Hz}$. A $250 \mathrm{~Hz}$ inhibitory input decreased the firing rate of the model neuron for all values of $\theta$. The maximum firing rate decreased to $31 \mathrm{~Hz}$, and the firing rate at $\theta= \pm 3$ decreased to $0.12 \mathrm{~Hz}$.

We scaled the tuning curves with modulatory input to best fit the baseline tuning curve (Fig. $4 B$ ). As in Figures 1 and 2, the tuning curves with modulatory input can be scaled to nearly fit the baseline curve. Parameters for Gaussian fits of the data in Figure $4 A$ are shown in Table 3 . These fits show that, in addition to the multiplicative scaling, there are systematic changes in the width of the tuning curve caused by modulatory input. Excitatory modulation caused an $8 \%$ increase in the width of the tuning curve. Inhibitory modulation caused a $6 \%$ decrease in the width of the tuning curve.
Table 3. Values of the fit parameters for the solid lines in Figure $4 A$ and the scale factors used in Figure $4 B$

\begin{tabular}{lllll}
\hline & $R_{\max }$ & $\sigma$ & $S$ & Scale factor \\
\hline Base & 41.0 & 0.622 & 0.508 & 1.00 \\
$250 \mathrm{~Hz}$ Exc & 54.3 & 0.669 & 1.14 & 1.39 \\
$250 \mathrm{~Hz} \operatorname{lnh}$ & 30.4 & 0.588 & 0.235 & 0.715 \\
\hline
\end{tabular}

Parameters are defined in Equation 6. Exc, Excitatory; Inh, inhibitory

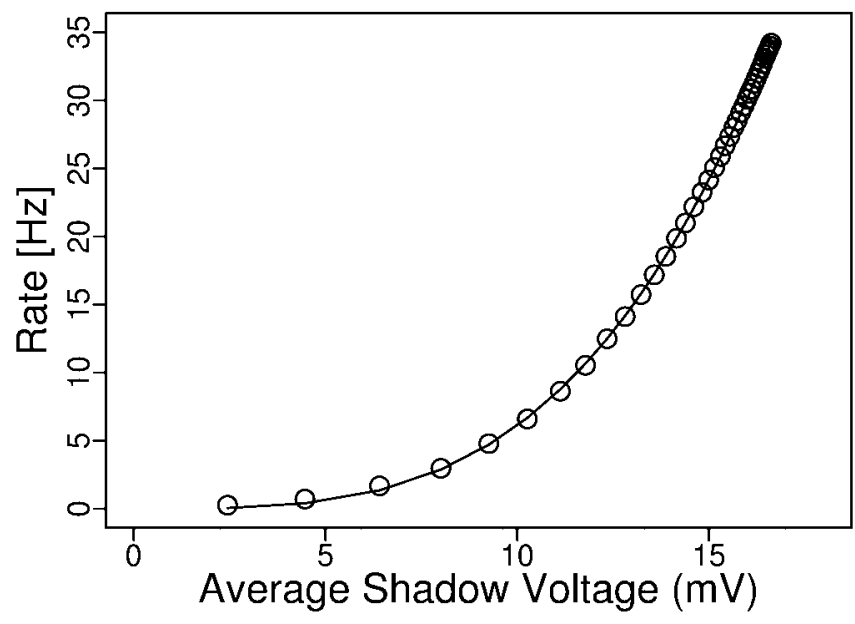

Figure 5. A plot of the average shadow voltage versus firing rate (circles) for the model neuron driven by an excitatory Poisson input (the same input as the Base curve in Fig. 1). Each data point represents an average of $2060 \mathrm{sec}$ trials. The voltage axis is shifted such that $V_{\text {rest }}$ is $0 \mathrm{mV}$. The solid line is a fit of $f=k V^{\alpha}$ to the data, with $k=0.0025 \mathrm{~Hz} /[\mathrm{mV}]^{\alpha}$ and $\alpha=3.4$. The shadow voltage is the voltage the neuron would have if spiking and postspiking reset were ignored (see Results).

Results obtained by injecting constant current, instead of adding a modulatory Poisson input, are very similar (data not shown). None of the modulatory inputs used in any of these simulations had an affect on the response variability of the model neuron as measured by the coefficient of variation of interspike intervals.

Although we have chosen the parameters for the integrateand-fire model carefully to match the experimentally measured properties of cortical neurons, our results do not depend on the detailed parameters of the simulations. For example, nothing qualitatively changes if the membrane time constant is doubled or halved (by correspondingly changing the capacitance) or if the stimulus-dependent Poisson input is replaced by an injected current (so long as the amplitude of the current remains the same nonlinear function of the stimulus parameter). The reason for this robustness is shown by the following simple analytical model, which shows the more general conditions required for these results to hold.

\section{Analytical model}

We express the state of the neuron in terms of the "shadow voltage," defined to be the voltage the neuron would have if it did not spike or undergo postspike voltage resets. The effect of the voltage noise in the model neuron is to make the firing rate $f$ of the neuron depend on its mean shadow voltage $V$ as a power law (Hansel and van Vreeswijk, 2002; Miller and Troyer, 2002) (Fig. 5):

$$
f=k V^{\alpha} \text {. }
$$



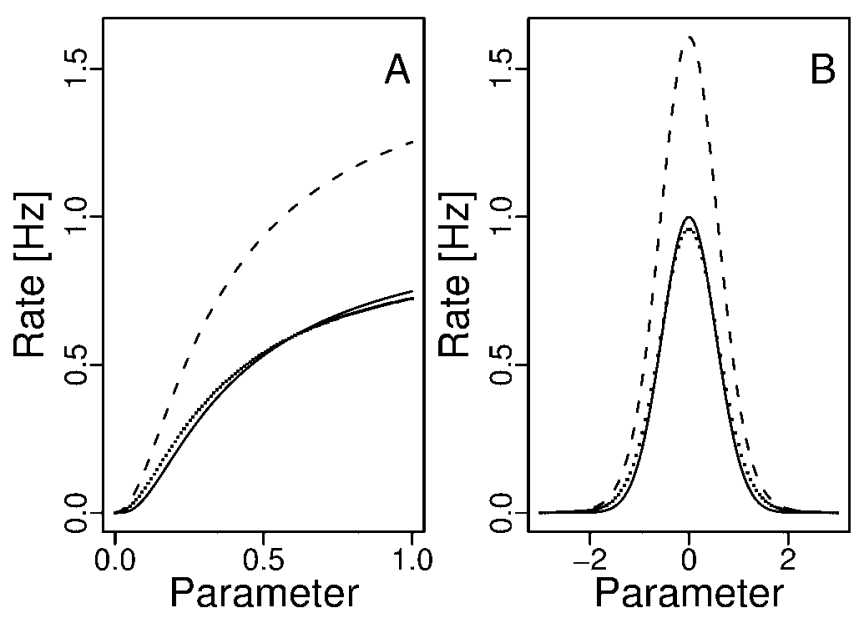

Figure 6. Plots of Equation 9 with $\alpha=3.4$ and $k=1.0 \mathrm{~Hz} /[\mathrm{mV}]^{\alpha}$. The solid lines correspond to $m=0 \mathrm{mV}$, and the dashed lines correspond to $m=0.15 \mathrm{mV}$. The dotted lines are the $m=0.15 \mathrm{mV}$ curves optimally (least squares) scaled to fit the $m=0 \mathrm{mV}$ curves. $A, d(p)$ is a hyperbolic ratio function with $C_{50}=0.133, n=1.2$, and $R_{\max }=1.0 \mathrm{mV} . B, d(p)$ is a Gaussian with $\sigma=1.0$ and an amplitude of $1.0 \mathrm{mV}$.

Here, $\alpha$ is $\sim 3.4$. Furthermore the shadow voltage is linear in the mean input, so it can be thought of as a function of a driving input and a modulatory input:

$$
V=d(p)+m .
$$

The modulatory input $m$ is a constant, whereas the driving input $d(p)$ is a function of an input parameter $p$. In our numerical simulations, $p$ corresponds to either contrast or the tuning parameter $\theta$. Likewise, $m$ corresponds to iontophoretic application of drugs, injected current, or modulatory synaptic inputs. Using these two equations, we obtain an expression for firing rate with respect to $d(p)$ and $m$ :

$$
f=k(d(p)+m)^{\alpha} .
$$

This equation is already sufficient to largely explain the results of our simulations. Letting $d(p)$ be a sigmoid (Fig. 6A) or a Gaussian (Fig. $6 B$ ), we can compare $k(d(p))^{\alpha}$ (Fig. 6, solid lines) with $k(d(p)+m)^{\alpha}$ for positive $m$, renormalized to best match $k(d(p))^{\alpha}$ (Fig. 6, dotted lines). Here, positive $m$ represents an excitatory modulatory input. We find that the effect of excitatory modulation is to approximately multiply a sigmoidal CR curve, but with a slight left shift of the curve (Fig. 6A), and to approximately multiply a Gaussian tuning curve, but with a slight widening of the curve (Fig. $6 \mathrm{~B}$ ). In both cases, the result of the simple model given by Equation 9 is essentially identical to the results of the integrate-and-fire simulations.

To further understand why Equation 9 leads to a gain change, we define the gain as the derivative of the firing rate with respect to the input parameter. Taking the derivative of Equation 9 with respect to $p$, we obtain an expression for the gain:

$$
\frac{\partial f}{\partial p}=k \alpha(d(p)+m)^{\alpha-1} \frac{\partial d(p)}{\partial p} .
$$

Because $\alpha>1$ the gain is directly related to the modulatory input, $m$.

However, Chance et al. (2002) point out that a change in $\frac{\partial f}{\partial p}$ for a given $p$ can arise from either a "true" gain change or a simple left or right shift of the nonlinear $f$ versus $p$ curve. To distinguish between these two cases, they plot $\frac{\partial f}{\partial p}$ versus $f$. Using Equation 9 we can rewrite Equation 10 as:

$$
\frac{\partial f}{\partial p}=k \alpha\left(\frac{f}{k}\right)^{\frac{\alpha-1}{\alpha}} \frac{\partial d(p)}{\partial p} .
$$

When $d(p)$ is linear in $p, \frac{\partial d(p)}{\partial p}$ is a constant. Therefore $\frac{\partial f}{\partial p}$ has no dependence on $m$, and changes in modulatory inputs $(m)$ do not affect the gain. This can be seen more directly from Equation 9: if $d(p)=a p+b$, then $f=k(a p+b+m)^{\alpha}$, so that $m$ simply left or right shifts the curve of $f$ versus $p$ without changing its shape. In the study by Chance et al. (2002), the driving input is an injected current and $d(p)$ is a linear function of $p$. In our results, and in many biologically relevant situations, $d(p)$ is not linear. In this case, $\frac{\partial d(p)}{\partial p}$ in Equation 11 is not constant but depends on $p$; when reexpressed in terms of $f$, there will also be a dependence on $m$. That is, the value of $\frac{\partial f}{\partial p}$ as a function of $f$ depends on $m$. Thus, changes in modulatory inputs do not simply shift the $f$ versus $p$ curve, they change its gain, its slope for a given value of $f$.

The gain changes observed in our numerical simulations are very nearly multiplicative; the slope at each point is changed by roughly the same factor. The above arguments explain why there should be a gain change but not why it should be nearly multiplicative. To make this more clear, it is useful to look at the expansion of Equation 9 to first order in $\frac{m}{d(p)}$ :

$$
k(d(p)+m)^{\alpha} \approx k\left(d(p)^{\alpha}+\alpha m d(p)^{\alpha-1}\right) .
$$

With no modulatory input, $m=0$, the firing rate is simply $k d(p)^{\alpha}$. If a non-zero modulatory input had a purely multiplicative effect on firing rate, we would expect it to add to the firing rate an amount proportional to $d(p)^{\alpha}$. Equation 12 shows that a non-zero $m$ actually adds an amount proportional to $d(p)^{\alpha-1}$. Thus, the multiplicative effects of modulatory inputs in our model depend on $d(p)^{\alpha-1}$ being similar in shape to $d(p)^{\alpha}$, which in turn depends on $\alpha$ being substantially larger than 1 . Of course, $d(p)^{\alpha}$ and $d(p)^{\alpha-1}$ cannot be perfectly identical in shape unless $d(p)$ is a constant, so small discrepancies from a perfectly multiplicative scaling are predicted; similar discrepancies are seen in some experimental results, as addressed in Discussion.

From the inputs represented in our simulations, the relationship between $d(p)^{\alpha}$ and $d(p)^{\alpha-1}$ is most easily seen when $d(p)$ is a Gaussian function. In this case, $d(p)^{\alpha}=\exp \left(-\alpha \frac{p^{2}}{2 \sigma^{2}}\right)$ and $d(p)^{\alpha-1}=\exp \left(-(\alpha-1) \frac{p^{2}}{2 \sigma^{2}}\right)$. So, when $m=0$, the firing rate of the neuron is a Gaussian, which is narrower than the Gaussian input $d(p)$ by a factor of $\sqrt{\alpha}$. A positive $m$ adds an amount proportional to a slightly wider Gaussian (narrower than the Gaussian input by a factor of $\sqrt{\alpha-1}$ ). This produces a new tuning curve that is multiplicatively scaled and wider than the curve with $m=0$ by an amount that depends on $m$ and $\alpha$. The width should increase by an amount no less than zero and no more than a factor of $\frac{\sqrt{\alpha}}{\sqrt{\alpha-1}}$. For $\alpha=3.4$, the width should increase no more than $19 \%$, which is consistent with results from the 

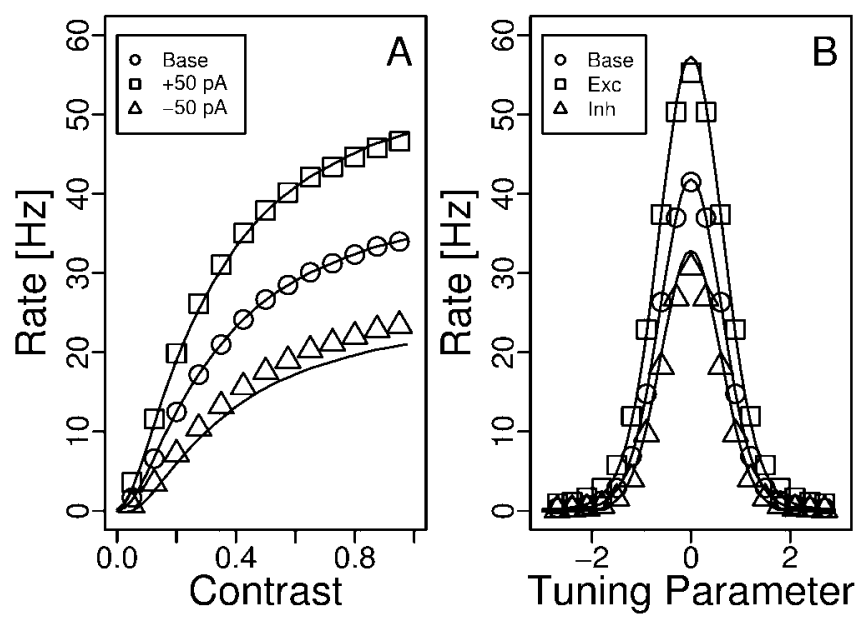

Figure 7. Comparison of predictions of Equation 12 (solid lines) to results of simulations (symbols). Solid lines are plots of the right side of Equation 12 using values for $d(p)$ and $m$ taken from the numerical simulations. The values of $K$ and $\alpha$ are the same as in Figure 5. $d(p)$ is the mean shadow voltage of the baseline curve without modulation. $m$ is the mean difference between the shadow voltage of the curve with modulation and the baseline curve. Symbols are the same as shown in Figures $2 A$ and $4 A . A, d(p)$ and $m$ taken from CR curves generated by numerical simulation (Fig. 2). $B, d(p)$ and $m$ taken from tuning curves generated by numerical simulation (Fig. 4). Circles, Tuning curves without modulatory input; squares, tuning curves with $50 \mathrm{pA}$ injected current $(A)$ or with $250 \mathrm{~Hz}$ excitatory Poisson input $(B)$; triangles, tuning curves with $-50 \mathrm{pA}$ injected current $(A)$ or $250 \mathrm{~Hz}$ inhibitory Poisson input $(B)$.

integrate-and-fire simulation in which modulatory excitation increased the width of the tuning curve by $8 \%$, consistent with the slight widening observed in Figure $6 B$.

The relationship between $d(p)^{\alpha}$ and $d(p)^{\alpha-1}$ is less clear when $d(p)$ is a hyperbolic ratio function. However, it is easy to show that if $d(p) \propto \frac{C^{n}}{C^{n}+C_{50}{ }^{n}}$, then $[d(p)]^{\alpha}$ reaches its halfmaximum at $C=\frac{C_{50}{ }^{n}}{\left[2 \frac{1}{\alpha}-1\right]^{\frac{1}{n}}}$, which increases with increasing $\alpha$. That is, $d(p)^{\alpha-1}$ should reach its half-maximum at a slightly lower value of $C$ than $d(p)^{\alpha}$, so that adding $d(p)^{\alpha-1}$ to $d(p)^{\alpha}$ should cause a slight left-shifting of the sigmoid curve. This is the result of excitatory modulation as seen in simulations and in Figure $6 \mathrm{~A}$.

The approximation in Equation 12 is justified in cases when $m / d(p)$ is small. Clearly, this is not always the case in our simulations; for instance, $d(p)$ goes to zero when the contrast is zero. In this case, the value of the function goes to $\mathrm{km}^{\alpha}$, and the relative error of the approximation is very large. However, as long as $m$ is sufficiently small, the absolute error is small and the approximation is useful. The usefulness of this approximation in describing our numerical results is shown in Fig. 7, which plots the right side of Equation 12 using values for $d(p)$ and $m$ taken from our integrate-and-fire simulations. $d(p)$ is simply the mean shadow voltage (without modulatory inputs) of the model neuron in the simulated CR and tuning curves. $m$ is the mean difference between the shadow voltage of the neuron with a modulatory input and the baseline. The firing rates reconstituted using Equation 12 (Fig. 7, solid lines) correspond well with the actual firing rates in the integrate-and-fire simulations (Fig. 7, symbols). This indicates that this approximation is a reasonable one for describing these simulations.

The analytical model assumes that inputs cause additive changes in the shadow voltage. This is true for input currents. However, input conductances need not translate additively into input currents. To completely describe the effects of an input conductance, one has to consider reversal potential effects by which the current flowing through a conductance depends not only on the size of the conductance but also on the driving force. As such, the analytical model does not completely account for the effects of conductances, particularly shunting inhibitory conductances with reversal potentials close to rest. Nonetheless, we have found that shunting $\left(\mathrm{GABA}_{\mathrm{A}}\right)$ conductances behave similarly to injected currents in our numerical model, causing multiplicative changes in the curve of stimulus parameter versus firing rate. This is probably because, over the range of shadow voltages for which the firing rate of the neuron is significantly different from zero, the changes in the driving force are relatively small. Similar arguments (Holt and Koch, 1997) lead to the result that shunting conductances, like injected currents, have an additive effect on the curve of input current versus firing rate (Holt and Koch, 1997; Chance et al., 2002).

We are arguing that the addition of two inputs, followed by raising to a power, gives an approximate multiplication. If the input-output function were an exponential (Gabbiani et al., 2002) rather than a power law, this relationship would be exact: $e^{a+b}=e^{a} e^{b}$. This raises the question of whether a better analytic approximation to our results might be given by an exponential rather than a power law. However, an exponential input-output relationship for the stimulus-induced firing rate must have the form $f=k\left(e^{V}-1\right)$ so that $f=0$ when $V=0$ (where 0 represents rest). We tried fitting a function of this form to the input-output relationship of the neuron but the fit is visibly considerably worse than that shown in Figure 5 for a power law. Furthermore the fits to the simulation data using this equation (equivalents of Figs. 6 and 7) are quite poor, particularly for excitatory modulatory input. We conclude that the power law gives the better description of our simulations.

The success of this simple analytical model in describing the more complex biophysical model used in simulations demonstrates the robustness of our results. The analytical model shows that achievement of multiplicative gain modulation depends on only two features of the biophysical model: the shadow voltage should be a roughly linear function of the mean input, and the output rate should be a power law of the shadow voltage with an exponent significantly larger than 1 . These are both attributes of a wide variety of biophysical models with a wide variety of parameters. In particular, the robustness with which noise induces a power law in a series of models has been demonstrated previously (Hansel and van Vreeswijk, 2002; Miller and Troyer, 2002), and we have also verified this: the power $\alpha=3.39$ found in our integrate-and-fire model becomes $\alpha=3.33$ if only AMPA and no NMDA is used for excitatory currents; $\alpha=4.06$ if the time constant is doubled; and $\alpha=3.16$ if the time constant is halved (the time constant was manipulated by changing the capacitance), with excellent fits of a power law in all cases. The change in $\alpha$ with a change in time constant is as expected theoretically: it has been shown that $\alpha$ should primarily depend on the distance from rest to threshold in units of the SD of the noise (Miller and Troyer, 2002); doubling the time constant decreases the noise and hence increases this distance, which increases $\alpha$, whereas halving the time constant increases the noise and hence decreases this distance, which decreases $\alpha$.

Do these two features hold for real cortical neurons? The assumption that the voltage of a neuron is approximately a linear sum of its inputs is often made, and, indeed, it is under this assumption that a mechanism of gain modulation by inhibition or excitation alone has proven elusive; if inputs can multiplica- 
tively influence one another, multiplicative gain modulations are likely to be easier to attain. Nonetheless, our model provides such a mechanism under the assumption of linear input summation. Much evidence exists that summation in cortical or hippocampal pyramidal neurons can be linear (Jagadeesh et al., 1993, 1997; Cash and Yuste, 1998, 1999). However, pyramidal neurons contain voltage-dependent conductances that can affect the summation of inputs (for review, see Reyes, 2001) and can cause inputs to summate in a nonlinear manner (Schwindt and Crill, 1998; Nettleton and Spain, 2000; Wei et al., 2001). In addition, dendritic integration can be nonlinear, although nonlinear conductances can correct this and linearize dendritic integration (Bernander et al., 1994; Cash and Yuste, 1998, 1999). A recent modeling study suggests that integration of multiple inputs on a single thin apical dendrite may be nonlinear but that integration between dendrites is remarkably linear (Poirazi et al., 2003a,b).

The second key assumption, of a power-law relationship between voltage and firing rate, seems likely to hold in many cortical neurons. This is a general outcome of the presence of neural noise in a variety of neural models (Hansel and van Vreeswijk, 2002; Miller and Troyer, 2002), and many studies indicate the presence of substantial voltage noise in cortex (Arieli et al., 1996; Paré et al., 1998; Azouz and Gray, 1999; Tsodyks et al., 1999; Anderson et al., 2000; Hô and Destexhe, 2000). More specific evidence is provided by the finding by Anderson et al. (2000) that voltage noise can transform contrast-invariant voltage tuning into contrastinvariant spiking tuning in visual cortical neurons. As shown by Miller and Troyer (2002), a power-law transformation from voltage to spiking rate is the only such transformation that can achieve this, thus indicating that such a transformation is found in visual cortical cells.

The above arguments suggest that the two assumptions of our model may hold in many cortical cells. To provide further evidence that our proposed mechanism of gain modulation can apply to real cortical neurons, we now present numerical simulations of a model that includes some of the more detailed biophysical properties of cortical pyramidal neurons.

\section{Hodgkin-Huxley simulations}

The Hodgkin-Huxley neuron model includes three potentially important conductances not present in our integrate-and-fire simulations: voltage-activated spiking conductances, a spike frequency adaptation conductance, and a hyperpolarizationactivated mixed cation conductance $\left(I_{\mathrm{h}}\right)$. To demonstrate that the mechanism of gain change we are proposing is valid in this neuron model, we first show that the mean voltage of the neuron is approximately a linear function of the input it receives and that the firing rate of the neuron is related to the mean voltage by a power law. We then generate CR and Gaussian tuning curves for the model neuron with and without modulatory inputs. These curves are generated in the same way as the curves in the integrate-and-fire simulations presented above, but for simplicity the synaptic inputs (both modulatory and driving inputs) have been replaced with constant excitatory $\left(E_{\text {rev }}=0 \mathrm{mV}\right)$ or inhibitory $\left(E_{\text {rev }}=-80 \mathrm{mV}\right)$ conductances. Replacing these inputs with injected currents yields very similar results (data not shown).

\section{Input-output relationship}

A power-law relationship between mean voltage and firing rate has been reported previously for a Hodgkin-Huxley-type model neuron (Hansel and van Vreeswijk, 2002) with a noisy membrane potential. We confirm that this relationship is also present in the
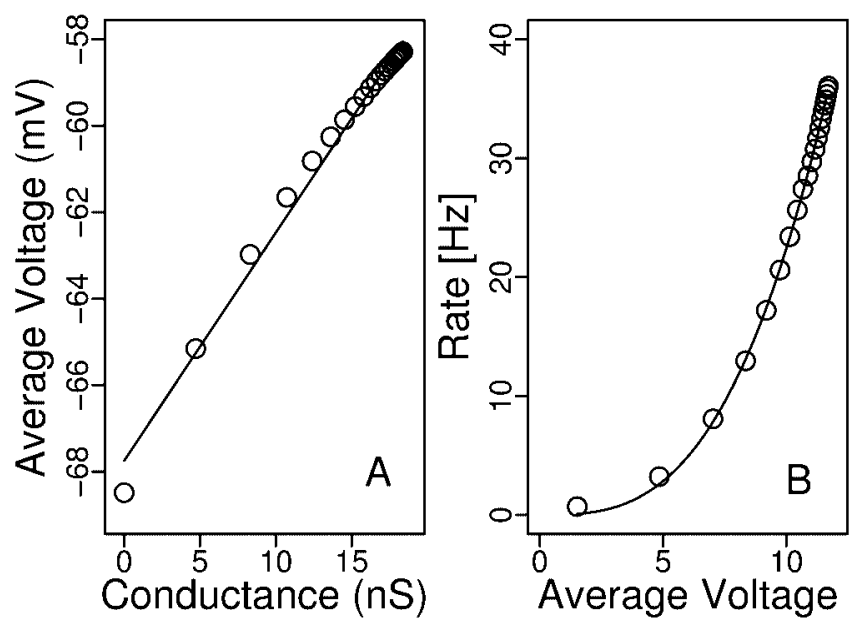

Figure 8. A, Plot of the input conductance versus average voltage (circles) for the HodgkinHuxley model neuron. The solid line is a linear fit to the data, with a slope of $0.528 \mathrm{mV} / \mathrm{nS}$ and an intercept of $-67.7 \mathrm{mV}$. B, Plot of the average voltage versus firing rate (circles) for the Hodgkin-Huxley model neuron. The voltage axis is shifted such that $V_{\text {rest }}$ is $0 \mathrm{mV}$. The solid line is a fit of $f=k V^{\alpha}$ to the data, with $k=0.024 \mathrm{~Hz} /[\mathrm{mV}]^{\alpha}$ and $\alpha=2.98$. In both $A$ and $B$, the input is the same as the Base curve in Figure 9 . Each data point represents an average of 10 trials, 5 sec each.

model neuron studied here (Fig. $8 B$ ). The mean voltage of the model neuron is related to the output firing rate by a power law with $\alpha=2.98$ and $k=0.024$. In addition we find that the relationship between excitatory input conductance and mean voltage is roughly linear over this range of output firing rate (Fig. $8 \mathrm{~A}$ ), although some deviation from linearity is seen as voltage approaches threshold.

\section{CR curve}

We construct a CR curve by introducing a stimulus-driven excitatory conductance, the magnitude of which is a hyperbolic ratio function of contrast with parameters $R_{\max }=20 \mathrm{nS}, C_{50}=0.133$, $n=1.2$, and $S=0$ (see Eq. 5). The modulatory inputs do not vary with contrast and are either a $3.5 \mathrm{nS}$ excitatory conductance or a $8.5 \mathrm{nS}$ inhibitory conductance.

Modulatory inputs have an effect on the CR curve very similar to that in the integrate-and-fire model (Fig. 9, compare Fig. 2). A $3.5 \mathrm{nS}$ excitatory conductance increased the maximum firing rate from 36 to $49 \mathrm{~Hz}$ and the firing rate at $\mathrm{C}=0.0$ from 0.7 to $2.3 \mathrm{~Hz}$. The average slope of the CR curve increased by $32 \%$. A $8.5 \mathrm{nS}$ inhibitory conductance decreased the maximum firing rate to 25 $\mathrm{Hz}$ and the firing rate at $\mathrm{C}=0.0$ to $0.18 \mathrm{~Hz}$. The average slope of the CR curve decreased by $30 \%$.

The changes in firing rate caused by the modulatory inputs are close to purely multiplicative gain changes (Fig. 9B). Deviations from purely multiplicative changes are indicated by the fit parameters in Table 4. Excitatory modulatory conductance shifted the baseline CR curve left, decreasing $C_{50}$ by $19 \%$. Inhibitory modulatory conductance shifted the curve right, increasing the baseline $C_{50}$ by $23 \%$.

\section{Tuning curve}

We construct a tuning curve by introducing a stimulus-driven excitatory conductance, the magnitude of which is a Gaussian function of a stimulus parameter $\theta$ with parameters $R_{\max }=17.5$ $\mathrm{nS}, \sigma=1.0$, and $S=0 \mathrm{~Hz}$ (see Eq. 6). The modulatory inputs were the same as for the CR curve, a $3.5 \mathrm{nS}$ excitatory conductance or an $8.5 \mathrm{nS}$ inhibitory conductance. 

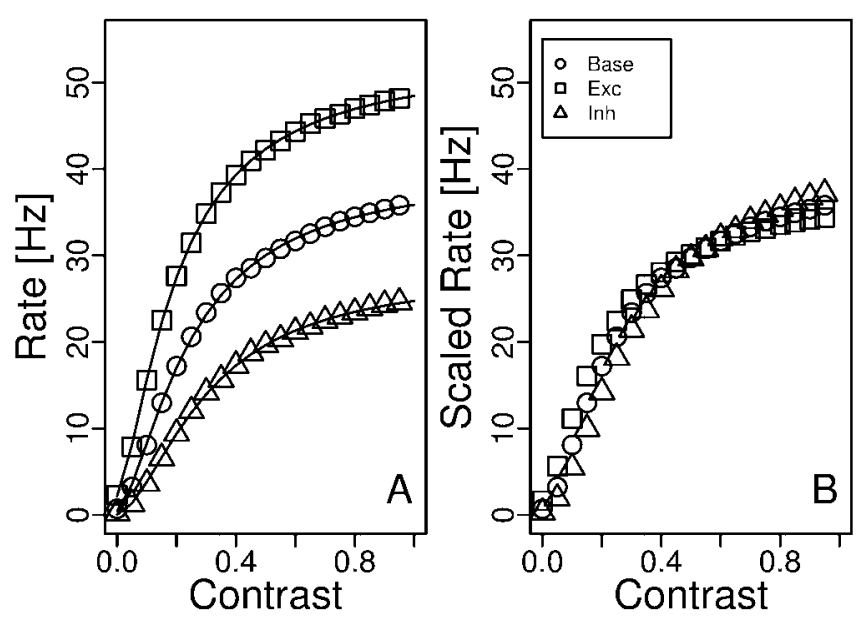

Figure 9. A, Plot of contrast versus firing rate for the Hodgkin-Huxley model neuron without modulatory input (circles) and with $3.5 \mathrm{nS}$ excitatory (squares) or $8.5 \mathrm{nS}$ inhibitory (triangles) modulatory conductances. Each data point represents an average of 10 trials, 5 sec each. Solid lines are fits of the data to a hyperbolic ratio function (Eq. 5). Parameters for the fits are shown in Table 4. B, Curves from $A$ scaled to optimally (least squares) fit the Base curve.

Table 4. Values of the fit parameters for the solid lines in Figure $9 A$ and the scale factors used in Figure $9 B$

\begin{tabular}{llllll}
\hline & $R_{\max }$ & $C_{50}$ & $n$ & $S$ & Scale \\
\hline Base & 39.48 & 0.2463 & 1.546 & 0.4358 & 1 \\
Exc & 50.71 & 0.2005 & 1.459 & 2.179 & 1.401 \\
Inh & 28.04 & 0.3022 & 1.677 & 0.02561 & 0.6615 \\
\hline
\end{tabular}

Parameters are defined in Equation 5. Exc, excitatory; Inh, inhibitory.
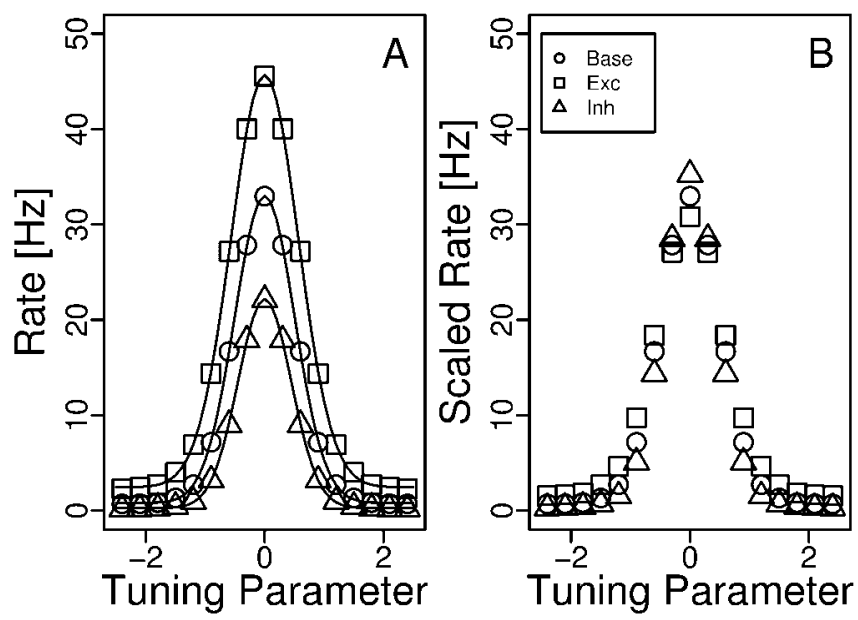

Figure 10. A, Plot of the tuning parameter in Equation 6 versus firing rate for the HodgkinHuxley model neuron without modulatory input (circles) and with $3.5 \mathrm{nS}$ excitatory (squares) or $8.5 \mathrm{nS}$ inhibitory (triangles) modulatory conductances. Each data point represents an average of 10 trials, 5 sec each. Solid lines are fits of the data to a Gaussian function (Eq. 6). Parameter values for the fits are shown in Table 5.B, Curves from $A$ scaled to optimally (least squares) fit the Base curve.

Modulatory inputs have an effect on the tuning curve very similar to that in the integrate-and-fire model (Fig. 10, compare Fig. 4). The driving input alone produced a maximum firing rate at $\theta=0.0$ of $33 \mathrm{~Hz}$ and a minimum firing rate at $\theta= \pm 2.5$ of 0.7 $\mathrm{Hz}$. We then added modulatory inputs to the neuron. The excitatory modulatory input increased the firing rate of the neuron for all values of $\theta$. The maximum response increased to $46 \mathrm{~Hz}$, and the firing rate at $\theta= \pm 2.5$ increased to $2.3 \mathrm{~Hz}$. The inhibitory
Table 5. Values of the fit parameters for the solid lines in Figure 10A and the scale factors used in Figure $10 B$

\begin{tabular}{lllll}
\hline & $R_{\max }$ & $\sigma$ & $S$ & Scale factor \\
\hline Base & 32.19 & 0.5044 & 0.7883 & 1 \\
Exc & 43.21 & 0.5644 & 2.44 & 1.478 \\
Inh & 21.91 & 0.4474 & 0.2701 & 0.6271 \\
\hline
\end{tabular}

Parameters are defined in Equation 6. Exc, Excitatory; Inh, inhibitory.

modulatory input decreased the firing rate of the model neuron for all values of $\theta$. The maximum firing rate decreased to $22 \mathrm{~Hz}$, and the firing rate at $\theta= \pm 2.5$ decreased to $0.18 \mathrm{~Hz}$.

The changes in firing rate caused by modulatory inputs are close to purely multiplicative gain changes (Fig. $10 \mathrm{~B}$ ). Deviations from purely multiplicative changes are indicated by the fit parameters in Table 5. An excitatory modulatory conductance caused a $12 \%$ increase in tuning curve width, whereas an inhibitory modulatory conductance caused an $11 \%$ decrease in width.

For both the CR curve and the tuning curve, deviations from purely multiplicative changes are in the same direction as the integrate-and-fire model and are similar in magnitude (although somewhat larger), which suggests that our proposed mechanism of gain change also operates in this more detailed biophysical model.

\section{Discussion}

The results demonstrate that changes in excitatory or inhibitory inputs alone can approximately multiplicatively change the gain of a neuron's response to a stimulus-dependent input. These gain changes observed in our model are primarily dependent on two things: an expansive nonlinearity (a power law with exponent substantially larger than one) relating the average membrane potential and the firing rate of the neuron, and an appropriately nonlinear dependence (e.g., sigmoidal or Gaussian) of the stimulus-dependent input rate on the corresponding stimulus parameter. Power-law input-output functions are likely to be ubiquitous in cortex because of voltage noise (Arieli et al., 1996; Paré et al., 1998; Azouz and Gray, 1999; Tsodyks et al., 1999; Anderson et al., 2000; Hô and Destexhe, 2000; Hansel and van Vreeswijk, 2002; Miller and Troyer, 2002), and cortical firing rates commonly have a sigmoidal or approximately Gaussian dependence on stimulus parameters. Hence, multiplicative gain changes should be common in cortex, and convergent inputs onto a neuron should multiplicatively modulate one another's gain, so long as one input (the "modulatory" input) is small relative to the peak input evoked by the other. This seems to be a natural result of the properties of cortical neurons and the input that they receive and could help explain the ubiquity of such gain changes observed experimentally (Andersen et al., 1985; Galletti and Battaglini, 1989; Boussaoud et al., 1993; Bremmer et al., 1997a; McAdams and Maunsell, 1999a,b; Treue and MartinezTrujillo, 1999; Trotter and Celebrini, 1999; Salinas and Thier, 2000).

\section{Predictions}

The most obvious prediction of our model is that at least some of the multiplicative gain changes observed in cortex will be found to arise from purely excitatory or purely inhibitory modulation (or more generally, from unbalanced modulatory inputs yielding a net excitation or inhibition). In addition, our work suggests a number of clues that would be consistent with a mechanism involving net excitation or inhibition.

Net excitatory or inhibitory modulatory inputs should cause 
small systematic deviations from a purely multiplicative gain change: gain increases should lead to decreases in $C_{50}$ and increases in tuning width, whereas gain decreases should yield opposite changes. However, the predicted deviations are small $(\sim 10-20 \%)$ and may not be observable in practice. Even so, this is a fundamental prediction of our model and may become more experimentally accessible in the future. Furthermore, the size of the deviations should be proportional to the size of the gain change, so larger changes in gain should give larger deviations from multiplication. This has two important implications. First, the deviations may become observable with sufficiently large gain changes. Second, modulation by unbalanced inputs should yield a correlation between the change in the amplitude of a tuning curve and the change in its width (for Gaussian tuning curves) or its $C_{50}$ (for sigmoidal tuning curves).

We also predict that multiplicative gain changes in vivo induced by unbalanced inputs should be accompanied by an upward or downward shift in mean voltage, but little change in total conductance or response variability. Such gain changes should occur in neurons that have substantial voltage noise and, thus, have power-law input-output functions. If the gain change is caused by a tonic excitation or inhibition (rather than a modulatory excitation or inhibition that only accompanies stimulusinduced input), then spontaneous activity levels should be modulated to the same degree as stimulus-induced responses.

\section{Previous theory and experiment}

We predict that tuning curves in cortex measured during intracellular current injection will exhibit multiplicative gain changes similar to those in our simulations. A gain decrease in the CR functions of neurons in primary visual cortex has been observed after injection of hyperpolarizing current (Fig. 3) (Sanchez-Vives et al., 2000). Accompanying this gain decrease was a statistically significant increase in the parameter $C_{50}$ describing the curve (Eq. 5), as predicted by our model.

Fox et al. (1990) examined the effects of externally applied glutamate receptor-binding drugs on the responses of cortical neurons to visual stimulation. They found that NMDA increased the gain of the CR curve of the neuron, whereas quisqualate shifted the curve upward. Our model suggests that simple depolarization induced by NMDA application, rather than nonlinearities in NMDA-induced responses, caused the gain increase. The seemingly straightforward effects of quisqualate are more difficult to explain. They may have been confounded by the fact that quisqualate has a number of effects besides activating nonNMDA ionotropic glutamate receptors, including binding to metabotropic glutamate receptors (Pin and Duvoisin, 1995; Chu and Hablitz, 2000) and glutamate transporters (Chase et al., 2001). We predict that a selective agonist of AMPA receptors, such as AMPA, would also cause a gain change.

A model proposed by Fox and Daw (1992) to account for their experimental results assumes that quisqualate acts exclusively at ionotropic non-NMDA receptors and that the firing rate of the neuron is linearly related to its membrane potential. In this case, the quisqualate-induced shifts are easily explained. However, given a more realistic power-law relationship between membrane potential and firing rate, it is difficult to account for the shifts induced by quisqualate based on its ionotropic action alone. To explain the effects of NMDA, they assumed cooperativity in binding between externally applied NMDA and synaptic glutamate released during visual stimulation, which requires that NMDA and glutamate bind to a common set of receptors. This was not always the case in their experiments; some cells exhibited gain increases during NMDA application, but no significant gain change during application of APV (an antagonist of NMDA receptors), implying that both bound to nonvisual NMDA receptors. In light of this, depolarization seems a more plausible explanation for NMDA-induced gain changes.

Our model complements the recent model of Chance et al. (2002). They showed that a balanced change in inhibitory and excitatory inputs could cause a multiplicative gain change as assessed by a change in a curve of firing rate versus current of a neuron. We show that, when one instead considers the curve of firing rate versus stimulus parameter, simple excitation alone or inhibition alone is sufficient to produce a gain change. This gain change is primarily dependent on the hyperpolarization or depolarization induced by the modulatory input, which causes only small changes in the conductance of the cell. In contrast, the gain changes described by Chance et al. (2002) require a relatively large change in both current noise and total conductance. A balanced change in excitation and inhibition together, as described by Chance et al. (2002), also produces a gain change in our model (data not shown). We conclude that a wide range of modulatory inputs, balanced or unbalanced, should induce a multiplicative gain change.

In cortical areas in which eye position modulates neural responses to visual stimuli, some visually driven neurons can also be driven directly by eye position alone (Boussaoud et al., 1993; Squatrito and Maioli, 1996, 1997; Bremmer et al., 1997a,b). Our model explains both the modulatory and driving effects of eye position on these neurons as resulting from a single excitatory input. This requires that direct responses to eye position be small relative to visual responses in such neurons, which seems consistent with experiments (Bremmer et al., 1997a,b; Squatrito and Maioli, 1997). This suggests some advantage of our proposal over that of Chance et al. (2002), which seems to require two different types of eye-position-evoked inputs for such neurons: a set of balanced inhibitory and excitatory inputs that modulates the gain of visual responses and another excitatory input that drives direct responses to eye position.

Changes in attention have been shown to multiplicatively scale the orientation and direction tuning curves of cortical neurons (McAdams and Maunsell, 1999a,b; Treue and MartinezTrujillo, 1999). One interpretation of these experimental results is that the neurons are responding with increased gain to the same visually induced input, as in our model. However, more recent results (Reynolds et al., 2000; Martinez-Trujillo and Treue, 2002) show that attention can cause a shift, rather than a gain change, in the CR function of these neurons. This suggests that the effects of attention may represent an increase in the effective contrast of the stimulus and not a change in the response gain of the neuron.

Treue and Martinez-Trujillo (1999) observed a slight (8\%), but not statistically significant, widening of MT direction tuning curves during feature-based attention. This widening, if substantiated by more data, would be in accordance with our model, which predicts a widening of tuning curves with increasing gain (Fig. 4). Changes in attention do not seem to cause changes in the width of orientation tuning curves in area V4 (McAdams and Maunsell, 1999a). Furthermore, although attention multiplicatively scaled tuning curves in V4 (including responses at nonpreferred orientations), it did not systematically affect spontaneous activity in the absence of visual stimulation. A net excitatory modulatory input, if tonically active, would have scaled spontaneous activity in the same manner as stimulus-evoked activity. Thus, if one models the effects of attention as a gain modulation rather than an increase in effective stimulus contrast, the model 
of Chance et al. (2002) may better explain the results from area $\mathrm{V} 4$. However, the widening of tuning curves in MT with attention is more in accordance with unbalanced or purely excitatory modulation; it would be interesting to determine whether spontaneous activity is affected by attention in MT and to determine conclusively whether attention affects the width of direction tuning curves in this area.

Our proposal is similar in spirit to that of Gabbiani et al. (2002), who suggested that an insect neuron does multiplication by subtracting one input from another at the level of voltages and having an exponential input-output relationship (although they found that a power law fit their input-output relationship better than an exponential). However, it differs in being based on the properties of cortical neurons.

Since this work was completed, two papers have appeared that address the role of shunting inhibition in gain modulation. Both highlight the importance of voltage noise. Prescott and De Koninck (2003) showed in a modeling study that dendritic saturation of the excitatory input, along with voltage noise, could cause shunting inhibition to divisively alter firing rate. The effect of saturation seems similar to that of a nonlinear, sigmoidal relationship between stimulus parameter and input rate in our model. Mitchell and Silver (2003) studied a cerebellar granular neuron that received relatively few excitatory synaptic inputs, all of which had large unitary conductances. As a result, an increase in input rate caused a significant increase in voltage noise. This, in turn, caused shunting inhibition to have a partially divisive effect on the curve of input rate versus output rate. If voltage noise does not increase significantly with input rate, as in the present and most previous studies of gain modulation, then shunting inhibition causes a subtractive shift in this curve (Chance et al., 2002).

\section{Implications for neuronal computation}

The question of whether a single neuron can biophysically multiply its inputs has long been of interest to those concerned with the computational capabilities of single neurons (Torre and Poggio, 1978; Mel, 1993; Koch, 1998; Gabbiani et al., 2002). We are proposing that a cortical neuron that adds its inputs at the level of voltages, but raises this net input to a power significantly greater than one to produce an output, can effectively compute a multiplication of the inputs [or more strictly, of functions of the inputs: the output $R$ is given by $R \approx f\left(i_{1}\right) g\left(i_{2}\right)$, where $i_{2}$ and $i_{2}$ are the inputs and $f$ and $g$ are some functions]. Furthermore if the input voltages are nonlinear functions of a stimulus parameter, then this multiplication will not produce a mere left or right shift of the curve of output versus parameter. Multiplication computed in this manner is only approximate. The approximation is accurate, although small systematic differences remain, when the modulatory input is substantially smaller than the driving input over the range of the tuning curve in which the driving input produces substantial responses.

\section{Conclusion}

Given a few basic assumptions about the common properties of cortical neurons, specifically the nonlinear ways that their input firing rates depend on the properties of a stimulus and their output firing rates depend on their input, we have shown that it should be expected that a smaller input will multiplicatively modulate the gain of the response to a larger input. No special mechanisms are required to account for these multiplicative interactions. Although other mechanisms may also play a role in experimentally observed gain changes, we are proposing that multiplicative gain changes are a normal property of the cortex, the natural outcome of these simple attributes of cortical neurons.

\section{References}

Albrecht DG, Hamilton DB (1982) Striate cortex of monkey and cat: contrast response function. J Neurophysiol 48:217-237.

Andersen R, Mountcastle V (1983) The influence of the angle of gaze upon the excitability of the light-sensitive neurons of the posterior parietal cortex. J Neurosci 3:532-548.

Andersen R, Essick G, Siegel R (1985) Encoding of spatial location by posterior parietal neurons. Science 230:456-458.

Anderson JS, Lampl I, Gillespie D, Ferster D (2000) The contribution of noise to contrast invariance of orientation tuning in cat visual cortex. Science 290:1968-1972.

Arieli A, Sterkin A, Grinvald A, Aertsen A (1996) Dynamics of ongoing activity: explanation of the large variability in evoked cortical responses. Science 273:1868-1871.

Azouz R, Gray CM (1999) Cellular mechanisms contributing to response variability of cortical neurons. J Neurosci 19:2209-2223.

Benardo LS (1994) Separate activation of fast and slow inhibitory postsynaptic potentials in rat neocortex in vitro. J Physiol (Lond) 476:203-215.

Bernander O, Koch C, Douglas R (1994) Amplification and linearization of distal synaptic input to cortical pyramidal cells. J Neurophysiol 72:2743-2753.

Boussaoud D, Barth T, Wise S (1993) Effects of gaze on apparent visual responses of frontal cortex neurons. Exp Brain Res 93:423-434.

Bremmer F, Ilg U, Thiele A, Distler C, Hoffmann K (1997a) Eye position effects in monkey cortex. I. Visual- and pursuit-related activity in extrastriate areas MT and MST. J Neurophysiol 77:944-961.

Bremmer F, Distler C, Hoffmann K (1997b) Eye position effects in monkey cortex. II. Pursuit- and fixation-related activity in posterior parietal areas LIP and 7A. J Neurophysiol 77:962-977.

Carmignoto G, Vicini S (1992) Activity-dependent decrease in NMDA receptor responses during development of the visual cortex. Science 258:1007-1011.

Cash S, Yuste R (1998) Input summation by cultured pyramidal neurons is linear and position-independent. J Neurosci 18:10-15.

Cash S, Yuste R (1999) Linear summation of excitatory inputs by CAl pyramidal neurons. Neuron 22:383-394.

Cavanaugh J, Bair W, Movshon J (2002) Nature and interaction of signals from the receptive field center and surround in macaque V1 neurons. J Neurophysiol 88:2530-2546.

Chance F, Abbott L, Reyes A (2002) Gain modulation from background synaptic input. Neuron 35:773-782.

Chase L, Roon R, Wellman L, Beitz A, Koerner J (2001) L-Quisqualic acid transport into hippocampal neurons by a cystine-sensitive carrier is required for the induction of quisqualate sensitization. Neuroscience 106:287-301.

Chu Z, Hablitz J (2000) Quisqualate induces an inward current via mGluR activation in neocortical pyramidal neurons. Brain Res 879:88-92.

Connors BW, Malenka RC, Silva LR (1988) Two inhibitory postsynaptic potentials, and $\mathrm{GABA}_{\mathrm{A}}$ and $\mathrm{GABA}_{\mathrm{B}}$ receptor-mediated responses in neocortex of rat and cat. J Physiol (Lond) 406:443-468.

Crair MC, Malenka RC (1995) A critical period for long-term potentiation at thalamocortical synapses. Nature 375:325-328.

Destexhe A, Paré D (1999) Impact of network activity on the integrative properties of neocortical pyramidal neurons in vivo. J Neurophysiol 81:1531-1547.

Destexhe A, Rudolph M, Fellous J, Sejnowski T (2001) Fluctuating synaptic conductances recreate in vivo-like activity in neocortical neurons. Neuroscience 107:13-24.

Doiron B, Longtin A, Berman N, Maler L (2001) Subtractive and divisive inhibition: effect of voltage-dependent inhibitory conductances and noise. Neural Comput 13:227-248.

Fox K, Daw N (1992) A model for the action of NMDA conductances in the visual cortex. Neural Comput 4:59-83.

Fox K, Sato H, Daw N (1990) The effect of varying stimulus intensity on NMDA-receptor activity in cat visual cortex. J Neurophysiol 64:1413-1428.

Gabbiani F, Krapp H, Koch C, Laurent G (2002) Multiplicative computation in a visual neuron sensitive to looming. Nature 420:320-324. 
Galletti C, Battaglini P (1989) Gaze-dependent visual neurons in area V3A of monkey prestriate cortex. J Neurosci 9:1112-1125.

Hansel D, van Vreeswijk C (2002) How noise contributes to contrast invariance of orientation tuning in cat visual cortex. J Neurosci 22:5118-5128.

Hines M, Carnevale N (1997) The NEURON simulation environment. Neural Comput 9:1179-1209.

Hirsch JA, Alonso J-M, Reid RC, Martinez L (1998) Synaptic integration in striate cortical simple cells. J Neurosci 18:9517-9528.

Hô N, Destexhe A (2000) Synaptic background activity enhances the responsiveness of neocortical pyramidal neurons. J Neurophysiol 84:1488-1496.

Holt GR, Koch C (1997) Shunting inhibition does not have a divisive effect on firing rates. Neural Comput 9:1001-1013.

Jagadeesh B, Wheat H, Ferster D (1993) Linearity of summation of synaptic potentials underlying direction selectivity in simple cells of the cat visual cortex. Science 262:1901-1904.

Jagadeesh B, Wheat H, Kontsevich L, Tyler C, Ferster D (1997) Direction selectivity of synaptic potentials in simple cells of the cat visual cortex. J Neurophysiol 78:2772-2789.

Jahr CE, Stevens CF (1990) Voltage dependence of NMDA-activated macroscopic conductances predicted by single-channel kinetics. J Neurosci 10:3178-3182.

Koch C (1998) Biophysics of computation: information processing in single neurons. Oxford: Oxford UP.

Krukowski AE, Miller KD (2001) Thalamocortical NMDA conductances and intracortical inhibition can explain cortical temporal tuning. Nat Neurosci 4:424-430.

Lester RA, Clements JD, Westbrook GL, Jahr CE (1990) Channel kinetics determine the time course of NMDA receptor-mediated synaptic currents. Nature 346:565-567.

Martinez-Trujillo J, Treue S (2002) Attentional modulation strength in cortical area MT depends on stimulus contrast. Neuron 35:365-370.

McAdams C, Maunsell J (1999a) Effects of attention on orientation-tuning functions of single neurons in macaque cortical area V4. J Neurosci 19:431-441.

McAdams C, Maunsell J (1999b) Effects of attention on the reliability of individual neurons in monkey visual cortex. Neuron 23:765-773.

Mel BW (1993) Synaptic integration in an excitable dendritic tree. J Neurophysiol 70:1086-1101.

Migliore M (2003) On the integration of subthreshold inputs from perforant path and Schaffer collaterals in hippocampal CA1 pyramidal neurons. J Comput Neurosci 14:185-192.

Miller KD, Troyer TW (2002) Neural noise can explain expansive, powerlaw nonlinearities in neural response functions. J Neurophysiol 87:653-659.

Mitchell S, Silver R (2003) Shunting inhibition modulates neuronal gain during synaptic excitation. Neuron 38:433-445.

Muller J, Metha A, Krauskopf J, Lennie P (2003) Local signals from beyond the receptive fields of striate cortical neurons. J Neurophysiol 90:822-831.

Nettleton J, Spain W (2000) Linear to supralinear summation of AMPAmediated EPSPs in neocortical pyramidal neurons. J Neurophysiol 83:3310-3322.

Palmer L, Nafziger J (2002) Effects of surround motion on receptive-field gain and structure in area 17 of the cat. Vis Neurosci 19:335-353.

Palmer S, Miller K (2002) The effects of inhibitory gain and conductance fluctuations in a simple model for contrast invariant orientation tuning in cat V1. Soc Neurosci Abstr 28:456.1.

Paré D, Shink E, Gaudreau H, Destexhe A, Lang EJ (1998) Impact of spontaneous synaptic activity on the resting properties of cat neocortical pyramidal neurons in vivo. J Neurophysiol 79:1450-1460.

Pin J, Duvoisin R (1995) The metabotropic glutamate receptors: structure and functions. Neuropharmacology 34:1-26.

Poirazi P, Brannon T, Mel B (2003a) Arithmetic of subthreshold synaptic summation in a model CA1 pyramidal cell. Neuron 37:977-987.
Poirazi P, Brannon T, Mel B (2003b) Pyramidal neuron as two-layer neural network. Neuron 37:989-999.

Pouget A, Sejnowski TJ (1997) Spatial transformations in the parietal cortex using basis functions. J Cogn Neurosci 9:222-237.

Pouget A, Snyder L (2000) Computational approaches to sensorimotor transformations. Nat Neurosci [Suppl] 3:1192-1198.

Prescott S, De Koninck Y (2003) Gain control of firing rate by shunting inhibition: roles of synaptic noise and dendritic saturation. Proc Natl Acad Sci USA 100:2076-2081.

Reyes A (2001) Influence of dendritic conductances on the input-output properties of neurons. Annu Rev Neurosci 24:653-675.

Reynolds J, Pasternak T, Desimone R (2000) Attention increases sensitivity of V4 neurons. Neuron 26:703-714.

Salinas E, Abbott L (1995) Transfer of coded information from sensory to motor networks. J Neurosci 15:6461-6474.

Salinas E, Abbott L (1996) A model of multiplicative neural responses in parietal cortex. Proc Natl Acad Sci USA 93:11956-11961.

Salinas E, Abbott L (2001) Coordinate transformations in the visual system: how to generate gain fields and what to compute with them. Prog Brain Res 130:175-190.

Salinas E, Thier P (2000) Gain modulation: a major computational principle of the central nervous system. Neuron 27:15-21.

Sanchez-Vives MV, Nowak LG, McCormick DA (2000) Membrane mechanisms underlying contrast adaptation in cat area 17 in vivo. J Neurosci 20:4267-4285.

Schwindt P, Crill W (1998) Synaptically evoked dendritic action potentials in rat neocortical pyramidal neurons. J Neurophysiol 79:2432-2446.

Sclar G, Maunsell JH, Lennie P (1990) Coding of image contrast in central visual pathways of the macaque monkey. Vision Res 30:1-10.

Smith M, Nelson A, Du Lac S (2002) Regulation of firing response gain by calcium-dependent mechanisms in vestibular nucleus neurons. J Neurophysiol 87:2031-2042.

Squatrito S, Maioli M (1996) Gaze field properties of eye position neurones in areas MST and 7A of the macaque monkey. Vis Neurosci 13:385-398.

Squatrito S, Maioli M (1997) Encoding of smooth pursuit direction and eye position by neurons of area MSTd of macaque monkey. J Neurosci 17:3847-3860.

Srinivasan M, Bernard G (1976) A proposed mechanism for multiplication of neural signals. Biol Cybern 21:227-236.

Stuart G, Spruston N (1998) Determinants of voltage attenuation in neocortical pyramidal neuron dendrites. J Neurosci 18:3501-3510.

Torre V, Poggio T (1978) A synaptic mechanism possibly underlying directional selectivity to motion. Proc R Soc Lond Biol Sci 202:409-416.

Treue S, Martinez-Trujillo J (1999) Feature-based attention influences motion processing gain in macaque visual cortex. Nature 399:575-579.

Trotter Y, Celebrini S (1999) Gaze direction controls response gain in primary visual-cortex neurons. Nature 398:239-242.

Troyer TW, Miller KD (1997) Physiological gain leads to high ISI variability in a simple model of a cortical regular spiking cell. Neural Comput 9:971-983.

Troyer TW, Krukowski AE, Priebe NJ, Miller KD (1998) Contrast-invariant orientation tuning in cat visual cortex: feedforward tuning and correlation-based intracortical connectivity. J Neurosci 18:5908-5927.

Tsodyks MV, Kenet T, Grinvald A, Arieli A (1999) Linking spontaneous activity of single cortical neurons and the underlying functional architecture. Science 286:1943-1946.

Wei D, Mei Y, Bagal A, Kao J, Thompson S, Tang C (2001) Compartmentalized and binary behavior of terminal dendrites in hippocampal pyramidal neurons. Science 293:2272-2275.

Zipser D, Andersen RA (1988) A back-propagation programmed network that simulates response properties of a subset of posterior parietal neurons. Nature 331:679-684. 